\title{
Actitudes del alumnado universitario ante la comunidad: Conciencia de ciudadanía y ApS
}

\author{
Belén Zayas Latorre, María Jesús Martínez-Usarralde \\ Universitat de València, España
}

\section{Resumen}

La formación en el diseño y ejecución de programas de servicio como el ApS ofrece oportunidades para que el alumnado incorpore la responsabilidad social y moral en el desarrollo de conocimientos, destrezas y valores necesarios para la toma de decisiones que afectan a las acciones individuales y colectivas relacionadas con cuestiones como los derechos humanos, la infancia o el desarrollo sostenible. Además, ayuda a expresar y debatir las argumentaciones, retos y aspectos críticos con sus iguales, así como a respetar aquellas opiniones y actos que no comparten. Desde esta investigación se pretende analizar si el alumnado universitario tiene conciencia de sí mismo/a como ciudadano/a, así como escrutar en qué medida el ApS desarrolla contenidos, estrategias y actitudes necesarios para la toma de decisiones que afectan a las acciones individuales y colectivas relacionadas con la comunidad.

\section{Palabras clave}

Aprendizaje servicio, responsabilidad social, comunidad, ciudadanía, compromiso.

Fecha de recepción: 9/II/2017

Fecha de aceptación: 9/III/2017 


\title{
Service learning and the attitudes of university students to the community
}

\begin{abstract}
Training in the design and implementation of service learning programmes offers opportunities for students to incorporate social and moral responsibility in the development of knowledge, skills and values required for making decisions that affect the individual and collective actions on issues such as human rights, children and sustainable development. In addition, it helps students to express and discuss arguments, challenges and critical issues with their peers, as well as to respect those opinions and acts that do not share. From this research is intended to analyze if university students have self-awareness as citizens, as well as scrutinizing how service learning develops content, strategies and attitudes necessary for making decisions that affect individual and collective actions related to the community.
\end{abstract}

\section{Keywords}

Service learning, Social Responsibility, Community, Citizenship, Engagement. 


\section{Introducción}

Vivimos en una sociedad en la que, lamentablemente, la falta de sensibilidad social forma parte de la cultura individualista del mundo postmoderno. Autores como García López, Gozálvez, Vázquez y Escámez (2010) defienden que la participación responsable en la sociedad civil exige una ciudadanía formada en competencias necesarias para demandar razones al gobierno acerca de sus políticas públicas y de legitimidad de las leyes, frente a la ley del silencio ante lo implícitamente aceptado por todos que se convierte en habitual. La participación responsable en la sociedad civil exige la preparación de la ciudadanía para que soliciten al gobierno las razones o justificación de sus políticas públicas y de legitimidad de las leyes.

A ello se refiere Cortina (1998) como códigos rojos, es decir, la "costumbre comúnmente aceptada, que es sin duda ley no escrita, pero la verdaderamente eficaz; a esos hábitos del corazón, que es preciso conocer para comprender lo que ocurre en una sociedad o en un segmento de ella" (Cortina, 1998, 38). Por ello, desde esta investigación, planteamos la necesidad de educar en la sensibilidad social al alumnado universitario, puesto que, dada su juventud, todavía no ha interiorizado esos códigos rojos que forman parte de nuestra sociedad. No sólo son los futuros profesionales de nuestra sociedad, sino que en ellos se confía la oportunidad de un cambio social, iniciándose desde la sensibilidad social.
Frente a esta realidad, se precisa un hueco de acción dentro del escenario de las competencias. En este sentido, García López (2010) plantea que las competencias entendidas como saber hacer parten de la relevancia otorgada al desempeño en el aprendizaje. En términos, precisamente, de aprendizaje, la capacidad se entiende como el potencial y se refiere al conjunto de recursos cerebrales básicos de los cuales puede hacer uso una persona, en las condiciones ambientales apropiadas, para construir funciones y comportamientos. La tercera concepción de las competencias relaciona las dos anteriores, refiriéndose a la idea de las primeras entendidas desde el enfoque competencia-desempeño.

Desde el acercamiento particular de García López, Gozálvez, Vázquez y Escámez (2010) al concepto de las competencias en la educación, clarificamos seis características fundamentales con las cuales nos sentimos identificadas y defenderemos en adelante: las competencias son aprendizajes adquiridos en contexto; las competencias son aprendizajes de carácter complejo; las competencias se manifiestan en desempeños; las competencias son evaluables; las competencias apuntan a la transversalidad $y$, por último, las competencias requieren del aprendizaje durante toda la vida.

Desde este planteamiento, se presenta, en este artículo, un marco teórico que sustenta la investigación, el planteamiento metodológico utilizado, los resultados obtenidos, así como las 
conclusiones de mayor relevancia.

\section{Marco teórico}

La formación de competencias para la participación social implica, entre otros aspectos, alfabetizar políticamente al alumnado, en sensibilidad social, en responsabilidad moral ante los problemas de injusticia social atendiendo al principio de beneficencia y en participación social en la universidad.

Las expectativas que se espera cumplir a través de la dimensión de alfabetización política se relacionan con la necesidad de desarrollar los conocimientos, destrezas y actitudes que el estudiantado necesita como futuro ciudadano y miembro de una sociedad global. En definitiva, una conciencia de lo público nacida desde la profunda reflexión y dominio del conocimiento a la que Barrio $(2015,21)$ se refiere "estudiar en serio y pensar sobre lo estudiado". Apostamos con ello por remarcar el valor del conocimiento para que el alumnado profundice sobre los derechos humanos, conozca las características de las instituciones claves como el parlamento y otras formas de gobierno, el sistema electoral y la importancia del voto, la importancia estratégica de los media en la sociedad, el mundo como una comunidad global, sus implicaciones políticas y el rol de la Unión Europea, y un largo etc.

Balbo (2008) defiende que la redefinición de la universidad implica el cambio de la personalidad de cada uno de sus actores, con altas competencias vinculadas a su profesión, a las que se suma un componente humanístico (arte, ética y filosofía) que le permitan desempeñarse como personas autorrealizadas y con sensibilidad social.

Con tal de abordar una modesta reflexión acerca de la rotura de las formas reproductoras y legitimadoras del saber positivista, hacemos alusión al término empleado por Cortina (1998), hábitos del corazón. A ellos se refiere como "la suma de las disposiciones morales e intelectuales de los hombres de una sociedad, incluyendo en ellas la conciencia, la cultura y las prácticas diarias" (Cortina, 1998, 36). La falta de sensibilidad social forma parte de los hábitos de nuestro corazón. Los códigos rojos de los que habla Cortina son los que imposibilitan la interiorización de la sensibilidad social. La autora hace referencia a estos hábitos como una ley del silencio ante lo implícitamente aceptado por todas las personas que acaba convirtiéndose en habitual.

Frente a esta realidad, a través de la responsabilidad social y moral, el alumnado desarrollará el conocimiento, destrezas y valores necesarios para la toma de decisiones que afectan a las acciones individuales y colectivas relacionadas con cuestiones como los derechos humanos, los derechos del niño o el desarrollo sostenible. La incorporación de esta dimensión de naturaleza global ayuda al alumnado a expresar y debatir las argumentaciones, retos y aspectos críticos con sus iguales, así como a respetar aquellas opiniones y actos que no comparten.

Respecto a la participación social en la 
universidad, el fenómeno del abstencionismo en las elecciones universitarias, tal y como mantiene Martín (2007), tiene su origen no en los propios universitarios, sino en cómo se articulan los mecanismos de participación y representación de las pequeñas democracias que son las universidades.

\section{Cualquier propuesta sobre la} participación del estudiantado en la universidad exige conocer ciertos rasgos de la participación juvenil, como por ejemplo la baja implicación política de los jóvenes. Una publicación preparada con motivo de la Conferencia Ministerial de Budapest y Viena, planteaba:

"El problema más comúnmente señalado con respecto a la participación de los estudiantes dentro de los países implicados en el proceso de Bolonia es el punto de vista cada vez más frecuente de que los estudiantes son consumidores, en lugar de miembros activos e iguales de la comunidad. Al considerar a los estudiantes de esta manera, las universidades fracasan en su función de crear ciudadanos activos y democráticos" (Trilla, Jover, MartínezMartí, Romañá, 2010, 2).

Los autores plantean diferentes tipos genéricos de participación, como son la participación simple, la participación consultiva, la participación proyectiva y la metaparticipación. Tanto la participación simple como la participación consultiva se caracterizan por disponer cierta connotación de exterioridad, ya que el sujeto es destinatario de una actividad u opina sobre ella, mientras que el desarrollo o ejecución del proyecto está en otras manos.
Respecto a la participación proyectiva, el participante se convierte en agente, y esta condición de agente es la que, según los autores, mejor caracteriza a la forma de participación. Ello requiere mayor compromiso y corresponsabilidad, por lo que es más intensa y compleja que las anteriores, ya que el participante es protagonista en la propia definición del proyecto, en la determinación de su sentido y de sus objetivos, en su diseño, planificación y preparación, en la gestión, ejecución y control del proceso.

\section{Metodología}

En la presente investigación se ha utilizado una metodología mixta, con técnicas cualitativas y cuantitativas, sumándose a estudios actuales en los que se mide el impacto del ApS en titulaciones de educación de diversas universidades españolas (Aznar y Belmonte, 2015; Capella, Gil, Martí y Chiva, 2015; Torío y García-Pérez, 2015).

El estudio cuantitativo permite un enfoque empírico-analítico mediante un pre-test y un post-test, cuya intervención consta en la participación en un proyecto de ApS, siendo la variable dependiente la actitud del alumnado universitario ante la comunidad. El estudio cualitativo supone el análisis hermenéuticointerpretativo que posibilita comprender los significados e intenciones de las acciones, comportamientos o decisiones de las personas a través de la interpretación de los discursos, como resultado de la interacción con los demás y el entorno, en este caso, 
mediante un grupo de discusión.

La muestra está formada por 60 alumnos de tercer curso, de los cuales 20 pertenecen al Grado de Pedagogía y 40 pertenecen al Grado de Educación Social. El procedimiento de muestreo utilizado ha sido invitar a participar al alumnado de las asignaturas de Filosofía de la Educación y Educación Internacional que han participado en proyectos de ApS en entrevistas personales, y convocar al alumnado que durante el curso ha manifestado una mayor conciencia ética y compromiso social (siendo 7 pertenecientes al Grado de Pedagogía y 14 pertenecientes al Grado de Educación Social), a formar parte del grupo de discusión.

\section{Resultados}

El principal objetivo de esta investigación se ha centrado en analizar si el alumnado universitario tiene conciencia de sí mismo como ciudadano, y escrutar en qué medida el ApS desarrolla el conocimiento, destrezas y valores necesarios para la toma de decisiones que afectan a las acciones individuales y colectivas relacionadas con la comunidad.

La disparidad de resultados con los que nos encontramos pone en evidencia que el alumnado tenga una actitud definida ante la comunidad, pues hay Ítems que retroceden, otros que se mantienen y otros que mejoran.

Los Ítems que sufren un retroceso tras la intervención son aquellos que hacen referencia al papel del estudiante, a la obligación de no ignorar los problemas sociales, y a la dedicación del tiempo libre a ayudar a otras personas. Llama especialmente la atención este retroceso, incluso el elevado porcentaje de alumnado que se muestra indiferente ante las cuestiones planteadas. Ello puede ser debido a la concepción individualista que actualmente se tiene del mundo universitario, el exceso de tareas del estudiantado, la cultura institucional de trabajar por objetivos etc., son cuestiones que dificultan la implicación y compromiso social.

Referente a los que no han sufrido ningún cambio se debe a que, por un lado, existen ítems que en el pre-test han obtenido un porcentaje elevado como por ejemplo el ítem "Considero importante saber detectar los problemas que se dan en nuestra sociedad para poder mejorarla", con un $99,2 \%$. Hecho que demuestra que el alumnado universitario dispone de una alta conciencia por los problemas sociales ya antes de participar en el proyecto de ApS, y que tras la intervención la conciencia llega al 100\% del estudiantado. En cambio, el ítem "Me haría feliz colaborar en un proyecto colectivo para promover una mayor participación del alumnado universitario" no sólo no se produce un cambio sino que el porcentaje tanto en el pre-test como en el post-test gira en torno al 50\%. Ello puede ser debido al enfoque explícito de felicidad que contiene el ítem, es decir, la felicidad forma parte de la ética de máximos que se formula desde la intimidad de cada persona, y el hecho de colaborar en un proyecto colectivo que promueva la participación no tiene por qué 
identificarse con la ética de máximos del alumnado. El ítem "Entiende por ciudadano el que participa en la comunidad para mejorarla" obtiene un $83,6 \%$ en el pre-test frente a un 84,4 $\%$ en el post-test. $Y$ en cuanto al ítem "Ves en la universidad una oportunidad para desarrollar tu papel como ciudadano participativo" obtiene un $68,0 \%$ frente a un $68,9 \%$ en el posttest.

Y por último, resaltamos los ítems que sí han mejorado tras la intervención. El ítem que mayor cambio ha sufrido es el que hace mención a "Tener la oportunidad de recibir una educación superior me obliga a asumir la responsabilidad de trabajar para mejorar la sociedad" que manifiesta un aumento en el rango "bastante o mucho" de un 14,2\%. El ítem que en segundo lugar ha obtenido mejor puntuación en el post-test es el de "Las comunidades mejoran si participamos activa y desinteresadamente en ellas" habiendo aumentado en un 10,4\%. El Ítem "Trabajar por el bien común me satisface personalmente", aumenta su valoración positiva en un $4,9 \%$. En el Ítem "La formación universitaria promueve la crítica constructiva" se observa un cambio de un $67,2 \%$ a un $75,6 \%$, aunque lo más significativo es que en el pre-test un $22,7 \%$ de la muestra no contesta, y esta cifra desciende a un $12,2 \%$ en el post-test. $Y$ por último, el Ítem "La formación universitaria te ayuda a detectar los problemas reales que se dan en la sociedad actual" pasa de un $71,1 \%$ a un $78,9 \%$.

Los resultados obtenidos ponen de
RIDIS.

manifiesto que el ApS constituye una oportunidad para asumir la responsabilidad de trabajar por la mejora social, que es posible visibilizar la mejora a través de la participación activa y que produce una gran satisfacción personal en quien lo practica.

Así pues, podemos afirmar que las actitudes que tiene el alumnado universitario ante la comunidad vienen determinadas por la concepción que la institución universitaria transmite sobre sí misma, por la ética de mínimos referida a lo exigible por una mejor convivencia entre la ciudadanía, por la ética de máximos referida a la concepción de felicidad y por la oportunidad que ofrece la universidad para trabajar y experimentar la mejora social a través del ApS.

Respecto a si el estudiantado universitario considera que la universidad realmente potencia la ciudadanía, los resultados nos demuestran que el alumnado percibe la participación en un programa de ApS como una actividad ofrecida desde el aula que permite trabajar para el bien común, que promueve la crítica constructiva en su formación, y que se convierte en un instrumento que ayuda a detectar los problemas reales que se dan en la sociedad. En cambio, la intervención parece no ser suficiente para que el alumnado vea en la universidad una oportunidad para desarrollar su papel como ciudadano participativo.

El análisis correlacional referente a la percepción que tiene el alumnado 
acerca de si la universidad desarrolla la ciudadanía influye en su actitud ante la comunidad, expone que, a partir de la participación en un proyecto de ApS, el alumnado ha aumentado la conciencia de que su formación como futuro profesional ha de estar al servicio de la mejora social, lo que le obliga a adquirir una responsabilidad como ciudadano.

La ejecución de proyectos de ApS en la universidad produce en el estudiantado la percepción de que ésta promueve la crítica constructiva orientada a la mejora social, lo que le conduce a nuevos planteamientos de su actitud ante la comunidad. Es decir, si la universidad posibilita la realización de actividades que suponga trabajar para el bien común, favorece en el alumnado la conciencia de que su papel como estudiante no sólo se limita al estudio de materias, sino que el tener la oportunidad de recibir una educación superior, le obliga a asumir la responsabilidad de trabajar para mejorar la sociedad.

Este contexto provoca en el alumnado cierta predisposición a dedicar parte de su tiempo a buscar recursos que ayuden a personas vulnerables y excluidas, considera que tiene la obligación de no ignorar los problemas sociales, valora la importancia de saber detectar los problemas que se dan en la sociedad para poder mejorarla, asume una mayor responsabilidad ante la realización de proyectos para mejorar la sociedad, toma conciencia de la importancia de participar activamente. $Y$ no hay que olvidar que estas actitudes generan en el alumnado un sentimiento de felicidad por colaborar en proyectos de participación que transforman la realidad, tal y como se manifiesta en los resultados obtenidos del grupo de discusión (figura 1).

\begin{tabular}{|l|l|}
\hline \multicolumn{1}{|c|}{ Categorías } & \\
\hline Satisfacción personal & $\begin{array}{l}95 \\
\%\end{array}$ \\
\hline $\begin{array}{l}\text { Aspectos referidos al desarrollo personal } \\
\text { y social }\end{array}$ & $\begin{array}{l}95 \\
\%\end{array}$ \\
\hline Compromiso profesional & $\begin{array}{l}83 \\
\%\end{array}$ \\
\hline Cualidades & $\begin{array}{l}80 \\
\%\end{array}$ \\
\hline Aprendizaje emocional & $\begin{array}{l}79 \\
\%\end{array}$ \\
\hline Aspectos referidos a la comunicación & $\begin{array}{l}65 \\
\%\end{array}$ \\
\hline Aprendizaje teórico & $\begin{array}{l}46 \\
\%\end{array}$ \\
\hline Aspectos referidos al emprendizaje & $\begin{array}{l}35 \\
\%\end{array}$ \\
\hline Habilidades de y para la vida & $\begin{array}{l}25 \\
\%\end{array}$ \\
\hline Aspectos referidos a la sensibilización con & $\begin{array}{l}25 \\
\%\end{array}$ \\
\hline
\end{tabular}

Figura 1. Categorías obtenidas en el grupo de discusión y porcentajes según la importancia que le concede la muestra participante a cada una de ellas.

A modo de concusión, podemos afirmar que el ApS constituye una metodología mediante la cual no sólo se responde a las competencias transversales de la titulación de Pedagogía y de Educación 
Social, sino que además permite el aprendizaje y el ejercicio de la profesión creando las condiciones sociales que invitan al juicio, al sentimiento y a la conducta ética.

\section{Discusión y conclusiones}

A lo largo de la investigación se ha planteado qué papel desempeña o debería desempeñar la universidad en la formación ciudadana de los miembros de una sociedad. Esteban (2004) plantea que uno de los objetivos fundamentales de la universidad es formar personas y profesionales, es decir, preparar a los futuros profesionales para que comprendan las complejidades éticas que afectarán a su toma de decisiones en la práctica profesional así como a lo largo de su vida. En cambio, tal y como demuestran los resultados, si no existe una responsabilidad por parte de la institución y del profesorado, en asumir un aprendizaje ético de la profesión en la universidad, difícilmente podremos hablar de profesionalidad.

García López, Escámez y Jover (2010) hacen mención a la creación del Espacio Europeo de Educación Superior en la que la Conferencia de Ministros responsables de Enseñanza Superior (2003) se refiere a empleabilidad, explicitando la importancia de la enseñanza de los valores de la ética civil. La Conferencia de Ministros, celebrada en Bergen en mayo de 2005, volvió a insistir en la dimensión de la ciudadanía activa de la formación universitaria. En España, la Ley Orgánica 6/2001, de 21 de diciembre, de Universidades (LOU) modificada por la Ley Orgánica 4/2007 de 12 de abril, para adaptarse a la política de la Educación Superior europea, a partir de la cual se elabora un Estatuto del estudiante universitario y la creación del Consejo de estudiantes universitarios, se atiende a los derechos y deberes del estudiantado.

En este sentido, uno de los asuntos relevantes que debe asumir la universidad es preparar a los y las profesionales desde ciertos principios éticos, por lo que no debe limitarse a la transmisión del saber: debe generar opinión, demostrar su compromiso con el progreso social y erigirse en un ejemplo para su entorno.

Resulta obvio que en el marco normativo europeo en materia de educación superior, se apuesta y defiende la dimensión de una ciudadanía activa: incluso en España se ha avanzado en cuanto a la elaboración del Estatuto del Estudiante Universitario y la creación del Consejo de Estudiantes Universitarios. En cambio, los resultados de la investigación ponen de manifiesto que, para producir un cambio en la actitud del alumnado ante la comunidad, la universidad ha de convertirse en un espacio donde se desarrolle la ciudadanía. Y como plantean García López, Escámez y Jover (2010), éste constituye el principal reto actual de la educación superior: asumir plenamente la educación en valores de la ética civil como principio rector de la acción educativa y considerar las universidades como auténticos centros de ciudadanía.

Así pues, la presente investigación se 
suma a las ya realizadas (MartínezUsarralde, Zayas y Sahuquillo, 2016) y podemos afirmar que el desarrollo de la ciudadanía en la universidad influye de manera directa en la actitud del alumnado ante la comunidad, y que el ApS se erige en la herramienta que permite concebir la universidad como espacio de participación y de transformación social. Aunque no hay que olvidar que, para que el sello de identidad de la universidad sea el desarrollo profesional y ciudadano de su alumnado, se requieren políticas efectivas que se desarrollen desde una cultura cívica institucionalizada.

\section{Referencias bibliográficas}

Aznar, J.A. y Belmonte, L.J. (2015). Aprendizaje servicio y cooperación al desarrollo en la Universidad de Almería (España). Opción, Año 31, 74-92.

Balbo, J. (2008). La enseñanza de la ética a través de la inclusión de la responsabilidad social universitaria en el currículo. Revista educación en valores, 9, 99-107.

Barrio, J. Ma. (2015). La universidad en la encrucijada. En Gil, F. y Reyero, D. (Eds.) Educar en la universidad de hoy. Propuestas para la renovación de la vida universitaria. Madrid: Encuentro.

Capella, C., Gil, J., Martí, M. y Chiva, O. (2015). Estudio de caso múltiple con historias de vida en el grado de educación infantil: Aprendizaje-Servicio en la didáctica de la educación física.

Revista de Profesorado, 19, 1, 334-348.

Cortina, A. (1998). Hasta un pueblo de demonios. Ética pública y sociedad. Madrid: Taurus.

Esteban, F. (2004). Excelentes profesionales y comprometidos ciudadanos: un cambio de mirada desde la Universidad. Bilbao: Desclée de Brouwer.

García López, R. (2010). Proyecto Docente. Disciplina Actividad Docente: Obligatoria Filosofía de la educación. Valencia: Universitat de València.

García López, R., Escámez, J. y Jover, G. (2010). Ética profesional docente en la universidad. En García López, R., Escámez, J. y Jover, G. (Eds.) Ética profesional docente. Madrid: Síntesis.

García López, R., Gozálvez, V., Vázquez, V. y Escámez, J. (2010). Repensando la educación: cuestiones y debates para el siglo XXI. Valencia: Brief.

Ley Orgánica de Universidades (L.O.U.) Ley Orgánica 6/2001, de 21 de diciembre, de universidades. (BOE. núm. 307, de 24 de diciembre de 2001).

Martín, I. (2007). La participación política de los estudiantes universitarios dentro y fuera de la Universidad. Panorama Social, 6, 119-132.

Martínez-Usarralde, M.J., Zayas, B. y Sahuquillo, P.M. (2016). Aps y ciudadanía comprometida: aproximación descriptiva-exploratoria a un caso. Opción, 32 (8), 187-208.

Torío, S. y García-Pérez, O. (2015). Aprendizaje-Servicio, estrategia para la participación social en la Universidad. 
En Pérez Serrano, G. (Coord.),

Pedagogía social, universidad y

sociedad. Madrid: Universidad Nacional

de Educación a Distancia.

Trilla, J., Jover, G., Martínez, M. y Romañá, T. (2010). La participación de los estudiantes en el gobierno y la vida universitaria. En XXIX Seminario Interuniversitario de Teoría de la Educación. Formación y participación de los estudiantes en la universidad. Madrid: Universidad Complutense.

Nota

La presente investigación se ha llevado a cabo en el marco del Programa Estatal de I+D+i Orientada a los Retos de la Sociedad 2013 (Ministerio de Economía y Competitividad) "Aprendizaje-Servicio e Innovación en la Universidad. Un programa para la mejora del rendimiento académico y el capital social de los estudiantes" EDU2013-41687-R (BOE 1/08/2014). 